\title{
Fully Degradable Thioester-functional Homo- and Alternating Copolymers Prepared through Thiocarbonyl Addition-Ring- opening RAFT Radical Polymerization
}

Matt P. Spick, ${ }^{a}$ Nathaniel M. Bingham, ${ }^{a}$ Yuman Li, ${ }^{a}$ Janella de Jesus, ${ }^{a}$ Catia Costa, ${ }^{b}$ Melanie J. Bailey, ${ }^{\mathrm{a}}$ Peter J. Roth ${ }^{\mathrm{a}, *}$

${ }^{a}$ Department of Chemistry, University of Surrey, Guildford, Surrey, GU2 7XH, UK

${ }^{\mathrm{b}}$ Ion Beam Centre, University of Surrey, Guildford, Surrey, GU2 7XH, UK

* corresponding author email address p.roth@surrey.ac.uk 


\begin{abstract}
The radical ring-opening polymerization (RROP) of thionolactones provides access to thioester backbone-functional copolymers but has, to date, only been demonstrated on acrylic copolymers. Herein, the thionolactone dibenzo[c,e]oxepane-5-thione (DOT) was subjected to AIBN-initiated free radical homopolymerization which produced a thioester-functional homopolymer with a glass transition temperature of $95{ }^{\circ} \mathrm{C}$ and the ability to degrade exclusively into predetermined small molecules. However, the homopolymerization was impractically slow and precluded the introduction of functionality. Conversely, the RAFT-mediated copolymerization of DOT with $\mathrm{N}$ methylmaleimide (MeMI), $N$-phenylmaleimide (PhMI), and $N$-2,3,4,5,6pentafluorophenylmaleimide (PFPMI) rapidly produced well-defined copolymers with the tendency to form alternating sequences increasing in the order MeMI $\ll$ PhMI $<$ PFPMI, with estimated reactivity ratios of $r_{\mathrm{DOT}}=0.198$ and $r_{\mathrm{PFPMI}}=0.0078$ for the latter system. Interestingly, defects in the alternating structure were more likely caused by (degradable) DOT-DOT sequences rather than (non-degradable) MI-MI sequences, which was confirmed through paper spray mass spectrometric analysis of the products from aminolytic degradation. Upon the aminolysis of backbone thioesters, maleimide repeating units were ring-opened, forming bisamide structures. Conversely, copolymer degradation through a thiolate did not result in imide substitution but nucleophilic para-fluoro substitution on PFPMI comonomer units, indicating the ability of DOT-MI copolymers to degrade under different conditions and to form differently functional products. The RROP of thionolactones has distinct advantages over RROP of cyclic ketene acetals and is anticipated to find use in the development of well-defined degradable polymer materials.
\end{abstract}




\section{Introduction}

Degradable polymers, i.e. chains that contain easily cleavable linkages in the backbone, are promising materials for a variety of applications; ranging from a view to reduce microplastic pollution to biomedical applications such as tissue engineering and drug delivery. ${ }^{1-4}$ However, it remains a challenge to design polymers that have useful and tuneable properties while intact and, at the same time, the potential to degrade fully, i.e. exclusively into small molecules with predictable properties such as solubility, toxicity, and chemical behaviour. These requirements are essential for most applications.

Radical ring-opening polymerization (RROP) is an advantageous synthetic method to produce degradable polymers. It involves cyclic monomers that can add onto a chain end radical, ring-open, and install a cleavable group in the backbone. ${ }^{3,5}$ The literature on RROP is dominated by two classes of monomers: cyclic ketene acetals ${ }^{6}$ and allyl sulfide lactones. ${ }^{7-9}$ Both classes provide an ester backbone functionality. As a radical method, RROP is, in principle, compatible with the suite of reversible deactivation radical polymerization (RDRP) methods ${ }^{10,11}$ and the plethora of heterogeneous polymerization protocols, ${ }^{12,13}$ which provide control over polymer architecture and the ability to produce well-defined nano-materials. ${ }^{14-17}$ At the same time, the high tolerance of radical polymerization toward functional vinyl comonomers provides opportunities to design degradable polymers with desirable properties such as biocompatibility or stimulus-responsiveness. In contrast to these manifold options for tuning the behaviour of the intact polymers, controlling the size of the degradation fragments remains a difficult challenge in RROP. ${ }^{3}$

In principle, a homopolymer prepared from a RROP monomer will degrade into predetermined small molecules upon full degradation. However, not all RROP monomers can be homopolymerized into a well-defined material. With CKAs, ring-retaining 1,2-vinyl polymerization can be a substantial side reaction and produces repeat units with ketal side groups without backbone degradability. ${ }^{3,6}$ Additionally, radical ring-opening homopolymerization offers limited options for the inclusion of additional functionality which must be installed into the RROP monomer itself. In 
this context, it is noteworthy that a recent study into novel amino-functional CKAs did not find all novel species to (homo)polymerize. ${ }^{18}$ Allyl sulfide lactones, on the other hand, produce methylenefunctional repeat units and cause crosslinking in formulations containing more than $20 \%$ feed of the allyl sulfide lactone. This crosslinking precludes the preparation of soluble homopolymers. ${ }^{7}$

An advantageous situation would be a combination of a RROP monomer with a vinyl comonomer that results in a controlled and predictable incorporation of the degradable linkages along the polymer backbone with a well-defined (average) number of vinyl repeat groups between the cleavable sites. Unfortunately, the realisation is far from trivial as most radical copolymerizations involve disparate monomer reactivities and result in copolymers with a compositional gradient. ${ }^{19,20}$ Recently, Shi and Agarwal $^{21}$ and Hill and co-workers ${ }^{22,23}$ investigated the copolymerization of CKAs with $N$-functional maleimides. These electron-poor monomers are have been known to prefer alternating copolymerization ${ }^{24}$ with electron-rich comonomers, such as styrene, ${ }^{25,} 26$ although this behaviour is not apparent with most other vinyl comonomers. ${ }^{27}$ In combination with CKAs, $N$ functional maleimides had a preference to form alternating copolymers, which was attributed to a charge transfer from the electron-rich CKA to the electron-poor maleimide. ${ }^{22}$ Alternating polyester copolymers could further be tuned and functionalised by the use and post-polymerization modification of a cyanuric chloride-functional maleimide comonomer. ${ }^{23}$ Strictly alternating copolymers, $(\mathrm{AB})_{\mathrm{n}}$, are ideal materials to degrade exclusively into predetermined $A \mathrm{~B}^{\prime}$ fragments. However, ring-retaining vinyl polymerization resulted in the introduction of non-degradable repeat units. For the combination of $N$-ethylmaleimide with the CKA 2-methylene-4-phenyl-1,3dioxolane, an estimated 30 mol- $\%$ of CKAs polymerized with ring-retention, ${ }^{22}$ while the copolymerization of 2-methylene-1,3-dioxepane with $N$-phenylmaleimide involved 46-61\% ringretention when conducted at $120{ }^{\circ} \mathrm{C}$ and at least $94 \%$ ring-retention when conducted at $60{ }^{\circ} \mathrm{C} .{ }^{21}$ Conversely, complete ring-opening of the CKA 5,6-benzo-2-methylene-1,3-dioxepane was observed in the copolymerization with $N$-functional maleimides under dilute solution conditions at $80-90{ }^{\circ} \mathrm{C} .{ }^{23}$ 
More recently, Bingham and Roth $^{28}$ and, independently, Smith and co-workers ${ }^{29}$ developed thiocarbonyl addition-ring-opening (TARO) radical polymerization. Inspired by the RAFT process, this method involves the irreversible ring-opening of a thionolactone which, uniquely, provides a thioester backbone functionality. This method is advantageous because it proceeds under complete ring-opening and involves an easily-prepared thionolactone monomer, dibenzo[c,e]oxepane-5thione (DOT), that is stable under ambient conditions. ${ }^{28}$ Additionally, while displaying similar hydrolytic stability as oxoesters, thioesters offer additional selective pathways for degradation. To date, the copolymerization of DOT has only been demonstrated with a small subset of acrylic comonomers. These formulations did not show ideal copolymerization behaviour with thionolactones typically being incorporated at a higher rate than the acrylic comonomer. The homopolymerization of DOT was reported to be unsuccessful $\left(<2 \%\right.$ monomer conversion), ${ }^{28,29}$ which was attributed to retardation resulting from slow-ring-opening or termination reactions of the intermediate radical. $^{28}$

Herein, we present the first detailed description of a radically-made thioester backbone-functional homopolymer and show that it degraded into the expected repeat unit fragments. As the homopolymerization of DOT was, however, impractically slow, we investigated the copolymerization behaviour of DOT with six electron-deficient comonomers, maleic anhydride, fumaronitrile, diethyl fumarate, $N$-methylmaleimide, $N$-phenylmaleimide, and $N$-2,3,4,5,6pentafluorophenylmaleimide, of which the first three did not show any appreciable copolymerization. The selection of $N$-functional maleimides, however, copolymerized rapidly with DOT to form predominantly alternating copolymers with measured comonomer reactivity ratios similar to those reported for maleimide-CKA copolymerizations and with a tendency to form (degradable) DOT-DOT sequences rather than (non-degradable) maleimide-maleimide sequences. Degradation through aminolysis and thiolysis was investigated and the ability of the copolymers to degrade fully was confirmed through mass spectrometry. 


\section{Experimental Section}

Details on instrumentation and the synthesis of monomers and a RAFT agent are given in the supporting information.

DOT Homopolymerization. DOT (149 mg, $0.658 \mathrm{mmol}, 5.4$ eq.), DMSO (3.0 mL) and AIBN (20.2 mg, $0.123 \mathrm{mmol}, 1$ eq.) were mixed, sealed with a septum and degassed by bubbling nitrogen for $30 \mathrm{~min}$. The mixture was then heated to $60^{\circ} \mathrm{C}$ for 7 days before being cooled to RT and exposed to air, achieving a monomer conversion of $<10 \%$. The reaction mixture was further treated with AIBN ( 15 mg), degassed as before and heated to $60{ }^{\circ} \mathrm{C}$ for 3 days. This process was repeated a further five times, retreating with AIBN every 3-4 days. After a total of 27 days, an accumulated monomer conversion of $22 \%$ was reached and the polymer was purified by precipitation into diethyl ether (approximately 20-fold in volume) three times and the polymer was collected as a white solid by centrifugation followed by drying in a vacuum at room temperature. See supporting information (Figures S2-S10) and main text for spectroscopic analysis.

RAFT Copolymerization of DOT with three $N$-functional maleimides was done at comonomer feed ratios of $50 \%$ with the aim of forming alternating copolymers. DOT, $N$-functional maleimide, $S$-benzyl-S'-propyl trithiocarbonate (1 eq.), AIBN (0.25 eq.) and solvent (total monomer conc. $=1.55 \mathrm{M}$ ) were added into a ground-glass joint tube. For $N$-methylmaleimide DMSO was used as the solvent, whilst for $N$-phenylmaleimide acetonitrile was used to reduce an apparent side reaction which afforded a pink colouration when DMSO was used. For N-2,3,4,5,6pentafluorophenylmaleimide anisole was used as solvent which was a better solvent for DOT compared to acetonitrile. Mixtures were sealed with a rubber septum and degassed with nitrogen for 15 min through a needle, with a shorter needle fitted for gas release. The tube was then placed in a pre-heated oil bath set at $80{ }^{\circ} \mathrm{C}$ and left overnight (18 hours). After cooling and exposing to air, the monomer conversion was determined by ${ }^{1} \mathrm{H}$ NMR analysis. The crude polymer solution was purified by precipitation into petroleum ether (approximately 30-fold in volume) and the polymer was collected as an off-white solid by centrifugation followed by drying in a vacuum at room 
temperature. See supporting information (Figures S12-S20) and main text for spectroscopic analysis of products.

Reactivity ratios of DOT with maleimides were determined through AIBN-initiated free radical polymerization using naphthalene as an inert internal standard. DOT and $N$-functional maleimide (in varying molar ratios, 100 eq total), solvent $(0.6 \mathrm{~mL}$, DMSO-d 6 for $N$-methylmaleimide, acetonitrile- $\mathrm{d}_{3}$ for $N$-phenylmaleimide and $N$-2,3,4,5,6-pentafluorophenylmaleimide) and AIBN (1 eq) were mixed. Naphthalene (50 eq) was added. A pre-polymerization ${ }^{1} \mathrm{H}$ NMR spectrum was obtained as reference. The mixture was degassed by bubbling nitrogen for $15 \mathrm{~min}$. Due to their low volatility, loss of monomers during degassing was deemed insignificant. The mixtures were then heated to $80{ }^{\circ} \mathrm{C}$ for between five and fifteen minutes, keeping conversions as low as practicable. After cooling to RT, the mixtures were analysed by ${ }^{1} \mathrm{H}$ NMR spectroscopy. The conversions of monomers into polymer were estimated by comparing the integrals of residual monomers to the standard signal at $\delta / \mathrm{ppm}=7.90(\mathrm{dd}, 4 \mathrm{H}$ of naphthalene) before and after polymerization. The molar content of DOT in the copolymers was plotted over the molar DOT feed ratio and the data was fitted through least-squares non-linear regression using the Mayo-Lewis equation. ${ }^{28}$

RAFT Copolymerization Kinetics of DOT and $N$-methylmaleimide, as an example maleimide monomer, were determined through AIBN-initiated RAFT copolymerization. A stock solution was prepared containing DOT (345 mg, $1.53 \mathrm{mmol}, 50 \mathrm{eq}$ ), $N$-methylmaleimide (170 mg, $1.53 \mathrm{mmol}$, $50 \mathrm{eq}$ ), naphthalene (as internal standard, $150 \mathrm{mg}, 0.98 \mathrm{mmol}$ ), $S$-benzyl-S'-propyl trithiocarbonate (30.6 $\mu \mathrm{mol}, 1 \mathrm{eq})$, AIBN (7.7 $\mu \mathrm{mol} 0.25 \mathrm{eq})$, and DMSO-d 6 (4.0 mL). Aliquots of $0.4 \mathrm{~mL}$ each were taken from the stock solution, sealed with a septum and degassed by bubbling nitrogen for 15 min. Each aliquot was heated to $80{ }^{\circ} \mathrm{C}$ for a predetermined amount of time from 10 to 360 minutes. After cooling to RT, the mixtures were analysed by ${ }^{1} \mathrm{H}$ NMR spectroscopy and SEC. Monomer conversion was determined from comparison of signals of residual monomers with the standard.

Copolymer Degradation through Aminolysis. Copolymers (20 mg) were dissolved in THF (5 $\mathrm{mL})$ and isopropylamine $(2.5 \mathrm{~mL})$ was added. The mixtures were stirred at $\mathrm{RT}$ in a sealed vial 
overnight. Solvent and amine were evaporated by blowing in nitrogen gas followed by further evaporation in a vacuum oven for two days, and the residue was dissolved in THF and analysed by SEC, NMR and FT-IR spectroscopy (see main text and Figures S21-S24). For the degradation of DOT homopolymer, pDOT (1.8 mg) was dissolved in THF (2 mL) and isopropylamine $(1 \mathrm{~mL})$ was added. The mixture was stirred at RT for 4 days. After evaporation, the residue was analyzed by mass spectrometry, see Figure S11.

Polymer Degradation through Thiolysis with Concurrent para-fluoro Substitution. Propane-1thiol $(8 \mu \mathrm{L})$ and diazabicyclo[5.4.0]undec-7-ene (DBU, $12 \mu \mathrm{L})$ were dissolved in $\mathrm{CDCl}_{3}(1 \mathrm{~mL})$. Separately, p(DOT-alt-PFPMI) (20 mg) was dissolved in $\mathrm{CDCl}_{3}(1 \mathrm{~mL})$. The solutions were combined and stirred at RT. After $30 \mathrm{~min}$, a sample $(500 \mu \mathrm{L})$ was withdrawn and analysed by ${ }^{1} \mathrm{H}$ and ${ }^{19} \mathrm{~F}$ NMR spectroscopy. Complete disappearance of the broad polymer signals indicated complete degradation, see main text. 


\section{Results and Discussion}

DOT Homopolymerization

We recently presented a thiocarbonyl addition-ring-opening mechanism for the radical copolymerization of the thionolactone dibenzo[c,e]oxepane-5-thione (DOT, see Scheme 1A) into a thioester backbone-functional copolymer. An attempted free radical homopolymerization of DOT, however, failed, achieving $<1 \%$ monomer conversion after heating with AIBN in acetonitrile- $\mathrm{d}_{3}$ at $80{ }^{\circ} \mathrm{C}$ overnight. ${ }^{28}$ Experiments performed in the current work supported the proposed retardation but revealed that monomer conversions could be increased to up to $10 \%$ when using DMSO as solvent and larger amounts of AIBN with heating to $60{ }^{\circ} \mathrm{C}$ for several days. In order to produce a sufficient amount of DOT homopolymer, pDOT, the procedure was repeated by adding additional AIBN into the same vial (without removing the already formed polymer), degassing, and heating. After 27 days of repeated heating with AIBN, an accumulated monomer conversion of $22 \%$ was found by ${ }^{1} \mathrm{H}$ NMR spectroscopy and pDOT was isolated by precipitation into diethyl ether, Scheme 1A. This method was impractically slow and did not produce a well-defined polymer sample (the repeated re-initiation attempts likely lead to branching through chain transfer and the formation of side products through termination reactions). However, this reaction provided the opportunity to characterise, for the first time, a radically-prepared thioester backbone-functional homopolymer. Notably, this synthetic approach differed in mechanism, scope, and compatibility from the documented organocatalytic ring-opening homopolymerizations of $\varepsilon$-thiocaprolactone (wherein monomer and polymer feature a thioester, $\mathrm{C}(=\mathrm{O}) \mathrm{S})^{30}$ and of $\varepsilon$-thionocaprolactone (wherein monomer and polymer feature a thionoester, $\mathrm{C}(=\mathrm{S}) \mathrm{O}) .{ }^{31}$ To the best of our knowledge, pDOT-an unusual polymer featuring a biphenyl and a thioester in the backbone-had not previously been prepared and isolated by any method. The homopolymer was found to be soluble in DMSO, $\mathrm{CDCl}_{3}$, and THF, and insoluble in methanol, diethyl ether, and hexane. NMR spectroscopic analysis of pDOT through ${ }^{1} \mathrm{H}$ (Figure S2), ${ }^{1} \mathrm{H}-{ }^{1} \mathrm{H}$ COSY (Figure S3), ${ }^{13} \mathrm{C}$ (Figure S4-S5), ${ }^{1} \mathrm{H}-{ }^{13} \mathrm{C}$ HSQC (Figure S6-S7), ${ }^{1} \mathrm{H}-{ }^{13} \mathrm{C}$ HMBC (Figure S8-S9) and ${ }^{1} \mathrm{H}-{ }^{1} \mathrm{H}$ NOESY (Figure S10) methods allowed 
a complete assignment of all observed chemical shifts which proved invaluable in discerning DOTDOT linkages in copolymers (vide infra). The ${ }^{1} \mathrm{H}$ NMR spectrum indicated a ratio of approx. 6.5 DOT repeat units per cyanopropyl end group. Assuming termination by recombination (since termination by disproportionation is not possible on the involved $\mathrm{ArCH}_{2}$ radicals), this ratio referred to a number average degree of polymerization, DP = 13 and a calculated $M_{\mathrm{n}}^{\text {calc. }}$ of 3.1 $\mathrm{kg} / \mathrm{mol}$. The ${ }^{1} \mathrm{H}-{ }^{1} \mathrm{H}$ NOESY NMR spectrum showed that both hydrogens on the $\mathrm{Ar}-\mathrm{CH}_{2}-\mathrm{SC}(\mathrm{O}) \mathrm{Ar}$ units only interacted through space with one $\mathrm{H}$ atom on the same ring, but not with any $\mathrm{H}$ atoms on the neighbouring ring, suggesting (as to be expected from steric considerations) rotation along the Ar-Ar bond. The sample of pDOT had an SEC-measured PMMA-equivalent $M_{\mathrm{n}}{ }^{\mathrm{SEC}}$ of $4.9 \mathrm{~kg} / \mathrm{mol}$ with $Ð=M_{\mathrm{w}} / M_{\mathrm{n}}=2.0$ and a measured glass transition temperature of $T_{\mathrm{g}}=95^{\circ} \mathrm{C}$. Applying the Fox equation to $T_{\mathrm{g}} \mathrm{s}$ of DOT-tert-butyl acrylate copolymers published by Smith et al. ${ }^{29}$ gave calculated $T_{\mathrm{g}}$ of $107-111^{\circ} \mathrm{C}$ for $\mathrm{pDOT}$ (see Table $\mathrm{S} 1$ ), in general agreement with the above value, considering the low molar mass of our pDOT sample. An FT-IR spectrum of pDOT is shown and compared to copolymers in Figure 1. A sample of pDOT was treated with isopropylamine for degradation. Analysis of the product by mass spectrometry confirmed the formation of the expected $N$-isopropyl-2-(2-mercaptomethylphenyl)benzamide fragment and its disulfide (presumably formed through post-degradation oxidation), see Scheme 1A and Figure S11. 
Scheme 1. Radical ring-opening homopolymerization of dibenzo[c,e]oxepan-5-thione (DOT) and its degradation through aminolysis (A), structures of monomers from unsuccessful copolymerization with DOT (B), and RAFT radical copolymerization of DOT with three maleimides leading to predominantly alternating copolymers (C).
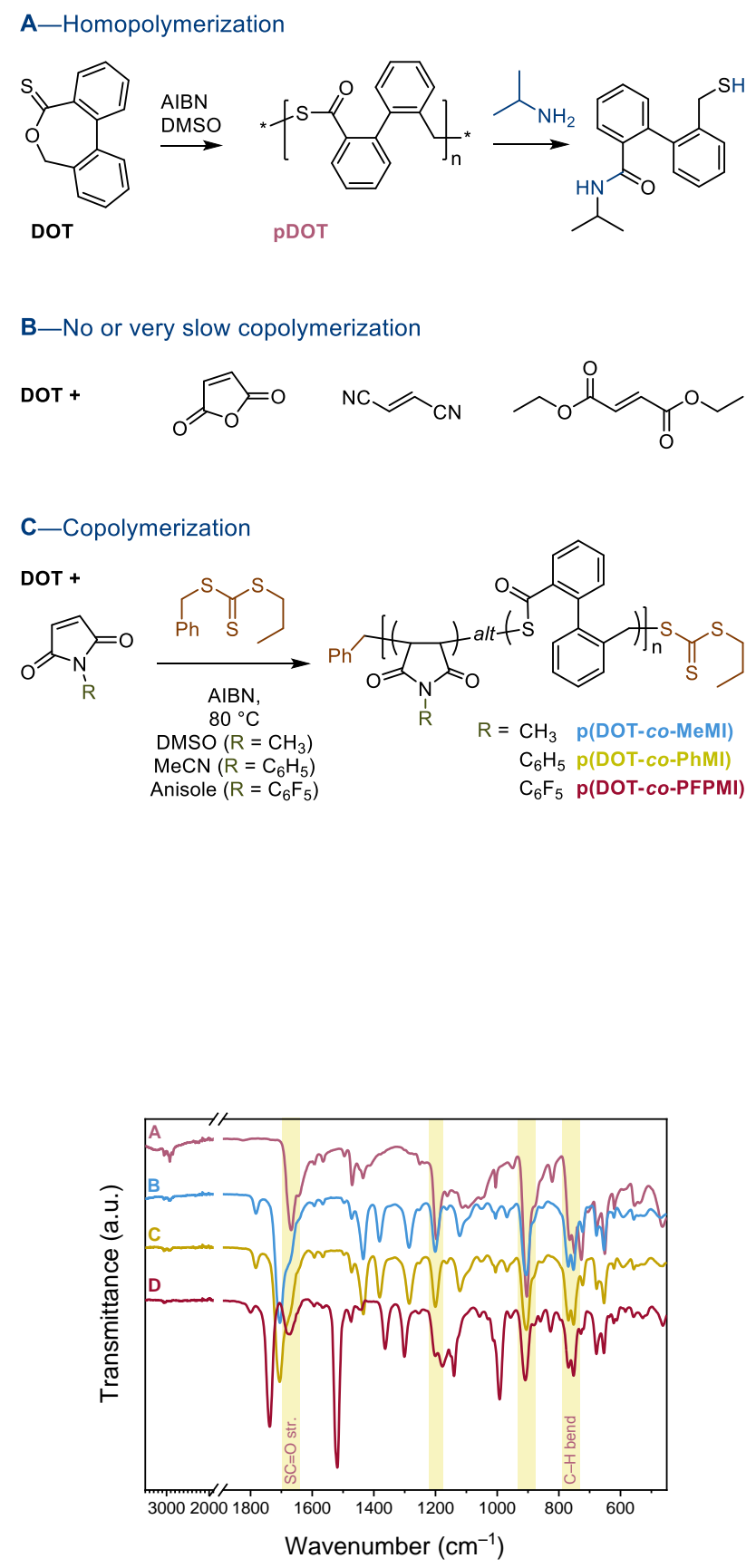

Figure 1. FT-IR spectra of pDOT homopolymer (A); p(DOT-alt-MeMI) (B); p(DOT-alt-PhMI) (C); and p(DOT-alt-PFPMI) (D) with characteristic vibrations of the homopolymer highlighted. 


\section{DOT Copolymerization}

Based on DOT homopolymerization being impractically slow and inspired by the alternating CKAmaleimide copolymers recently presented, ${ }^{22,23}$ we investigated the copolymerization behaviour of DOT with six electron-poor vinyl comonomers; maleic anhydride, fumaronitrile, diethyl fumarate, $N$-methylmaleimide $\quad$ (MeMI), $\quad N$-phenylmaleimide $\quad(P h M I), \quad$ and $\quad N-2,3,4,5,6-$ pentafluorophenylmaleimide (PFPMI), the latter of which was prepared in two steps (Scheme 1B,C). An attempted copolymerization of DOT with maleic anhydride resulted in the absence of polymerization, the observation of unassigned ${ }^{1} \mathrm{H}$ NMR signals, and brown discolouration and was not investigated further. Likewise, an attempted copolymerization of DOT with fumaronitrile gave no polymer. Diethylfumarate as comonomer gave global monomer conversions of $<10 \%$ in several attempts, which was deemed insufficient. Conversely, DOT copolymerized rapidly with all three $N$ functional maleimides in free radical copolymerizations and in AIBN-initiated RAFT copolymerizations in the presence of $S$-benzyl- $S^{\prime}$-propyl trithiocarbonate as a chain transfer agent, see Scheme 1C. RAFT copolymerization kinetics for a feed ratio of 50 equiv each of DOT and MeMI, as an exemplar maleimide, were determined in DMSO- $\mathrm{d}_{6}$ as solvent. The results indicated that global conversions of $>75 \%$ were reached after heating to $80{ }^{\circ} \mathrm{C}$ for $4 \mathrm{~h}$, Figure 2 . In this case, MeMI copolymerized slightly faster than DOT with the formed copolymers containing 53-59 mol$\%$ of MeMI repeat units and thus non-degradable maleimide-maleimide connections. Hill and coworkers made similar observations for 5,6-benzo-2-methylene-1,3-dioxepane- $N$-ethylmaleimide copolymers where defects in the alternating sequence were caused by a higher incorporation of maleimide units. ${ }^{23}$ The copolymerization of DOT-MeMI was well-controlled by the RAFT process with SEC indicating narrow monomodal traces with $Ð=M_{\mathrm{w}} / M_{\mathrm{n}}<1.20$ at all times and SECmeasured molar masses increasing with conversion, Figure 3. 


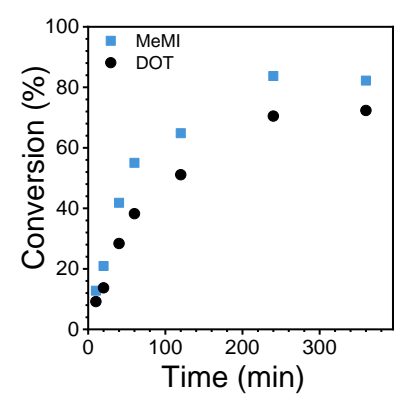

Figure 2. RAFT copolymerization kinetics of DOT-MeMI in a 50:50 feed in DMSO- $\mathrm{d}_{6}$ : conversion of each monomer (determined by ${ }^{1} \mathrm{H}$ NMR spectroscopy) vs time

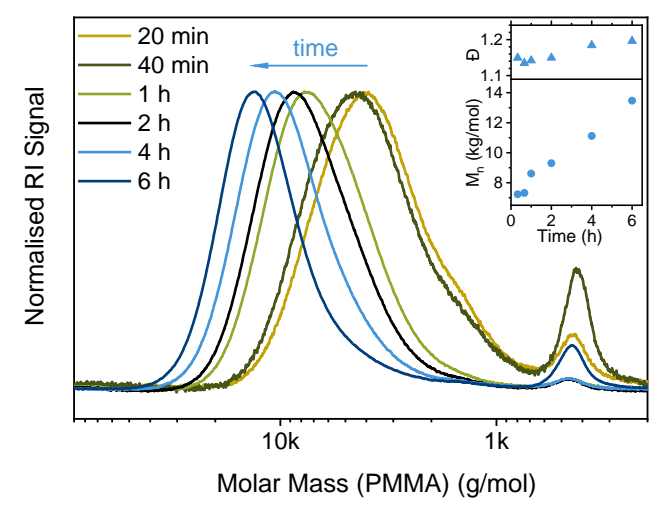

Figure 3. RAFT copolymerization kinetics of DOT-MeMI in a 50:50 feed in DMSO- $\mathrm{d}_{6}: \mathrm{SEC}$ chromatograms for reaction times from 20 min- $6 \mathrm{~h}$ and evolution of $M_{\mathrm{n}}^{\mathrm{SEC}}$ and $Ð$ with time (inset).

Copolymerizations of DOT with PhMI and PFPMI in DMSO yielded copolymers but accompanied by the formation of unidentified pink or red side products. Conversely, the copolymerization of DOT with PhMI and PFPMI in acetonitrile (and acetonitrile- $\mathrm{d}_{3}$ ) and anisole proceeded smoothly. The estimation of reactivity ratios, $r_{\mathrm{A}}$ and $r_{\mathrm{B}}$, is an expedient way to characterise the copolymerization behaviour of a comonomer combination $(\mathrm{A}, \mathrm{B})$. A reactivity ratio of $r_{\mathrm{A}}=1$ denotes no preference of a chain terminated in A to add another A monomer (homopropagation) or 
a B monomer (cross-propagation), while perfectly alternating copolymerization (exclusively crosspropagation) is found for the (theoretical and practically very rare) case of $r_{\mathrm{A}}=r_{\mathrm{B}}=0$. The observed values for the DOT-MeMI system of $r_{\mathrm{DOT}}=0.162$ and $r_{\mathrm{MeMI}}=0.654$ indicated a tendency of both comonomers to form alternating sequences but with non-negligible MeMI homopropagation events, see Figure 4A. These results agree with the faster incorporation of MeMI observed in the kinetic experiments described above. Gratifyingly, the situation was different for PhMI and PFPMI. For the DOT-PhMI system, $r_{\mathrm{DOT}}=0.348$ and $r_{\mathrm{PhMI}}=0.0136$ were determined, Figure $4 \mathrm{~B}$, and $r_{\text {DOT }}=0.198$ and $r_{\text {PFPI }}=0.0078$ for the combination of DOT with PFPMI, Figure 4C. These lower values were presumed to reflect steric and electronic effects of the $N$-aryl group lowering the propensity of the maleimide to homopolymerize. The slightly stronger tendency for the DOTPFPMI system to form alternating sequences was attributed to the electron-withdrawing effect of the perfluorinated aromatic and the resulting larger polarity difference of the comonomers during charge transfer polymerization. ${ }^{32}$ Notably, the estimated reactivity ratios for DOT-PhMI and DOTPFPMI were similar to those observed for alternating CKA-based copolymers, e.g. 2-methylene-4phenyl-1,3-dioxolane (MPDL)-N-ethylmaleimide $\left(r_{\text {MPDL }}=0.022 \text { and } r_{\text {PhMI }}=0.199\right)^{22}$ and 2methylene-1,3-dioxepane (MDO)-PhMI $\left(r_{\mathrm{MPDL}}=0.024\right.$ and $\left.r_{\mathrm{PhMI}}=0.120\right) .{ }^{21} \mathrm{~A}$ distinct difference, however, was that in the combination with DOT, the lower value of the reactivity ratio pairs (i.e., $r$ $\leq 0.02$ ) was found for the maleimide and not the cyclic RROP comonomer. This means that copolymers with a 50-50 comonomer composition were accessible at low DOT feed ratios up to $\sim 25$ mol-\% (see Figure 4B,C). For higher DOT feed ratios, defects in the alternating sequence arose chiefly from (degradable) DOT-DOT linkages, as opposed to (non-degradable) MI-MI connections — an advantage in the quest for fully degradable copolymers. 

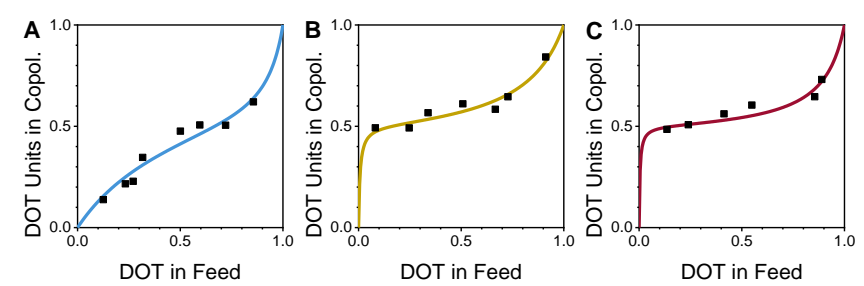

Figure 4. Molar DOT content in maleimide copolymer vs molar DOT fraction in the monomer feed with non-linear least-squares fitted curves for (A) $N$-methylmaleimide with $r_{\mathrm{DOT}}=0.162$ and $r_{\mathrm{MeMI}}=0.654$; (B) $N$-phenylmaleimide with $r_{\mathrm{DOT}}=0.348$ and $r_{\mathrm{PhMI}}=0.0136$; and (C) $N-2,3,4,5,6-$ pentafluorophenylmaleimide with $r_{\mathrm{DOT}}=0.198$ and $r_{\mathrm{PFPMI}}=0.0078$.

\section{Copolymer Characterization}

Following analysis of the copolymerization behaviour of DOT with $\mathrm{N}$-functional maleimides, three copolymers, $\mathrm{p}(\mathrm{DOT}$-alt-MeMI), $\mathrm{p}(\mathrm{DOT}$-alt-PhMI), and $\mathrm{p}(\mathrm{DOT}$-alt-PFPMI) were prepared with 50 mol-\% feed ratios of the comonomers and isolated by precipitation into petroleum spirit. The materials were characterised by FT-IR and NMR spectroscopy, DSC, and SEC. FT-IR spectra of the three copolymers are shown in Figure $1 \mathrm{~B}-\mathrm{D}$. The characteristic $\mathrm{SC}=\mathrm{O}$ stretching vibration of the DOT repeat units (seen for the pDOT homopolymer at $v=1656 \mathrm{~cm}^{-1}$ ) appeared as a shoulder on the imide band of the copolymers, except for p(DOT-alt-PFPMI), for which the imide band had shifted to higher wavenumbers and appeared as a separate band. ${ }^{1} \mathrm{H}-{ }^{13} \mathrm{C}$ HSCQ,${ }^{13} \mathrm{C}$, and ${ }^{1} \mathrm{H}-{ }^{13} \mathrm{C}$ HMBC NMR spectra for each copolymer are shown in Figures S12-20. The recording of 2-D spectra was necessary to appreciate the overlapping of $\mathrm{CH}_{2}$ and $\mathrm{CH}$ backbone resonances in the 2.6-4.0 ppm region of the ${ }^{1} \mathrm{H}$ spectra. Several points are worth making: DOT-DOT linkages, apparent through resonances of the $\mathrm{CH}_{2}$ group at $\delta_{\mathrm{H}}=4.05-3.62 \mathrm{ppm}, \delta_{\mathrm{C}}=31.6 \mathrm{ppm}$ in the HSCQ spectra, were observed for all three copolymers. MI-MI linkages, on the other hand, were not discernible, due to signals overlapping or too low signal intensity. The main feature of all copolymer NMR spectra were signals associated with alternating DOT-MI units: ${ }^{13} \mathrm{C}$ NMR spectra 
showed two imide carbonyl signals and two $\mathrm{CH}$ signals, suggesting different environments on either side of a MI repeat unit. ${ }^{1} \mathrm{H}^{-13} \mathrm{C}$ HMBQ spectra showed multiple bond correlations between neighbouring DOT and MI units, specifically, of MI backbone $\mathrm{CH}$ units with DOT $\mathrm{CH}_{2}$ groups and DOT thioester carbons. By integrating the ${ }^{1} \mathrm{H}$ NMR spectra, the molar compositions of the copolymers were estimated to be $\mathrm{p}\left(\mathrm{DOT}_{0.51}\right.$-alt-MeMI $\left.\mathrm{M}_{0.49}\right), \mathrm{p}\left(\mathrm{DOT}_{0.54}\right.$-alt-PhMI $\left.\mathrm{P}_{0.46}\right), \mathrm{p}\left(\mathrm{DOT}_{0.52}\right.$-alt$\mathrm{PFPMI}_{0.48}$ ), indicating a slightly higher DOT content for all three copolymers. Copolymer $\mathrm{p}\left(\mathrm{DOT}_{0.51}\right.$-alt-MeMI $\left.\mathrm{MI}_{0.49}\right)$ was presumed to have a slight compositional gradient starting with a MIenriched section (as argued above) and ending with DOT-enriched sequences due to this sample being taken to near-complete comonomer conversion. A composition gradient is to be expected for DOT-MI based on the estimated reactivity ratios being relatively large (see above). From the estimated molar compositions and from the integration of ${ }^{1} \mathrm{H}$ signals pertaining to DOT-DOT linkages, the molar percentage of non-degradable MI-MI connections were estimated to be $3.6 \%$ for DOT-MeMI, 3.0\% for DOT-PhMI, with the lowest value of 1.1\% estimated for DOT-PFPMI, in agreement with the above discussion. It should be noted, however, that these estimations are based on the integration of broad NMR resonances which are prone to relative errors of 10-20\%. The three copolymers had measured glass transition temperatures of $T_{\mathrm{g}}=151.4{ }^{\circ} \mathrm{C}(\mathrm{DOT}-\mathrm{MeMI})$, $156.4{ }^{\circ} \mathrm{C}(\mathrm{DOT}-\mathrm{PhMI})$, and $156.4{ }^{\circ} \mathrm{C}$ (DOT-PFPMI), showing very little (or no) dependence on the $N$-functionality, but a marked increase compared to DOT homopolymer. Polymaleimides are known to have high glass transition temperatures due to rigid backbones, with a documented $T_{\mathrm{g}}$ of MeMI homopolymer of $285{ }^{\circ} \mathrm{C} .{ }^{33}$ SEC-analysis, Figure 5, revealed dispersities of the copolymers between 1.29-1.56, higher than the kinetics samples discussed above, presumably because of longer reaction times and high conversions. 


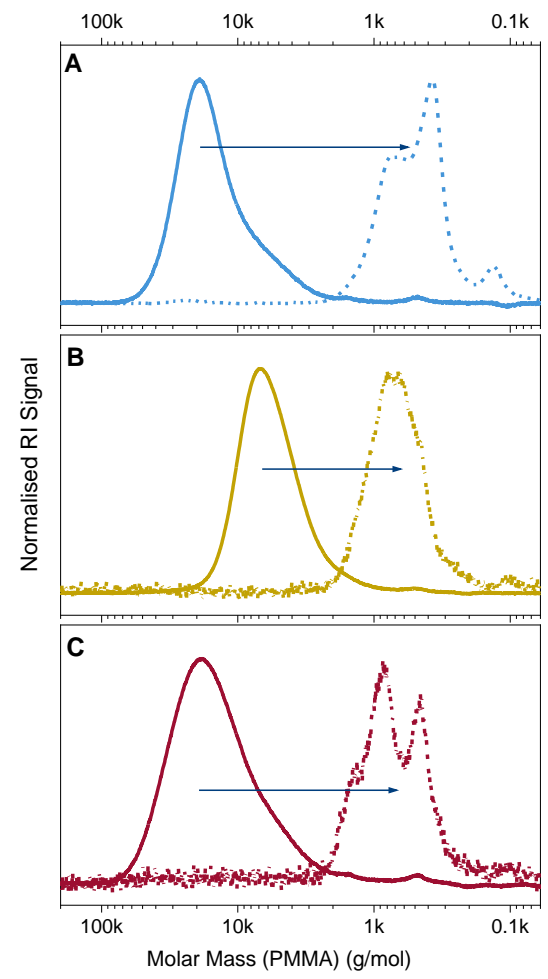

Figure 5. SEC traces of intact copolymers (solid lines) and after degradation with $N$ isopropylamine (dashed lines) for $(\mathrm{A}) \mathrm{p}(\mathrm{DOT}-$ alt $-\mathrm{MeMI})\left(M_{\mathrm{n}}^{\mathrm{SEC}}\right.$ (intact $)=12.6 \mathrm{~kg} / \mathrm{mol}, \mathrm{Đ}($ intact $)=$ $1.41, M_{\mathrm{n}}^{\mathrm{SEC}}($ degr. $)=0.41 \mathrm{~kg} / \mathrm{mol}, \mathrm{Ð}($ degr. $\left.)=1.42\right) ;(\mathrm{B}) \mathrm{p}(\mathrm{DOT}-$ alt $-\mathrm{PhMI})\left(M_{\mathrm{n}}^{\mathrm{SEC}}(\right.$ intact $)=5.1$ $\mathrm{kg} / \mathrm{mol}, \mathrm{Ð}($ intact $)=1.29, M_{\mathrm{n}}^{\mathrm{SEC}}($ degr. $)=0.62 \mathrm{~kg} / \mathrm{mol}, \mathrm{Ð}($ degr. $\left.)=1.23\right)$; and $(\mathrm{C}) \mathrm{p}(\mathrm{DOT}-$ alt PFPMI $\left(M_{\mathrm{n}}^{\mathrm{SEC}}(\right.$ intact $)=11.9 \mathrm{~kg} / \mathrm{mol}, Ð($ intact $)=1.56, M_{\mathrm{n}}^{\mathrm{SEC}}($ degr. $)=0.64 \mathrm{~kg} / \mathrm{mol}, Ð($ degr. $)=$ 1.30), showing the disappearance of intact polymers and formation of lower molar mass materials.

\section{Copolymer Degradation}

With three predominantly alternating thioester-functional degradable copolymers in hand, their degradation was investigated next. Copolymers were treated with an excess of $N$-isopropylamine in THF. After removing solvent and amine, the residues were analysed by SEC, NMR and FT-IR spectroscopy, and mass spectrometry. Unlike the copolymers, the degradation fragments were soluble in methanol, indicating a change in properties upon degradation. SEC traces indicated the complete disappearance of the intact copolymers and formation of materials of significantly lower 
(PMMA-equivalent) molar masses, Figure 5 (dashed lines). SEC traces of the degraded products were bi- or multimodal, reflecting the formation of species differing in the number of maleimide repeat units, aminolytic ring-opening of maleimide side groups (see below) and the potential formation of disulfides during sample preparation. ${ }^{1} \mathrm{H}$ NMR spectra of degraded samples showed sharp signals with similar chemical shifts as the intact copolymers, suggesting the formation of small molecules. In addition, ${ }^{1} \mathrm{H}$ NMR spectra of the degraded samples contained signals associated with isopropylamide groups, as to be expected for the aminolysis of thioesters, Figure S21. The most noticeable feature of FT-IR spectra, Figure S22-S23, was the change of the carbonyl stretching region, including the imide resonances. This suggested aminolytic cleavage also of the maleimide units and formation of two adjacent amide groups. Paper spray ${ }^{34}$ mass spectrometry confirmed the above conclusions. For all three copolymers, the fragment containing one DOT and one maleimide unit formed from alternating sequences was observed in positive and negative ions modes and detected as several ions. For all three copolymers, $N$-isopropyl-2-(2mercaptomethylphenyl)benzamide, the fragment resulting from the degradation of a DOT-DOT sequence (and the symmetrical disulfide) was observed, in agreement with the observation of these sequences by ${ }^{1} \mathrm{H}-{ }^{13} \mathrm{C}$ HSQC NMR spectroscopy. Disulfides containing maleimide units were only observed for the degradation of the MeMI copolymer. Aminolytic cleavage of maleimide units was found for all three copolymers with evidence of double amine attacks (imide cleavage and amide substitution with release of the $\mathrm{N}$-functional side group) found for the two aromatic maleimide systems, PhMI and PFPMI. These observations indicated that not only can degradable maleimide copolymers undergo cleavage into predictable small molecules but that the degraded fragments can react further. Fragments containing two MI units (providing evidence of non-degradable sequences) were found as positive ions for the MeMI and PhMI-containing copolymers but not for their PFPMI sister species, in agreement with the above description of the reactivity ratios, see Scheme 2 and Table 1. 

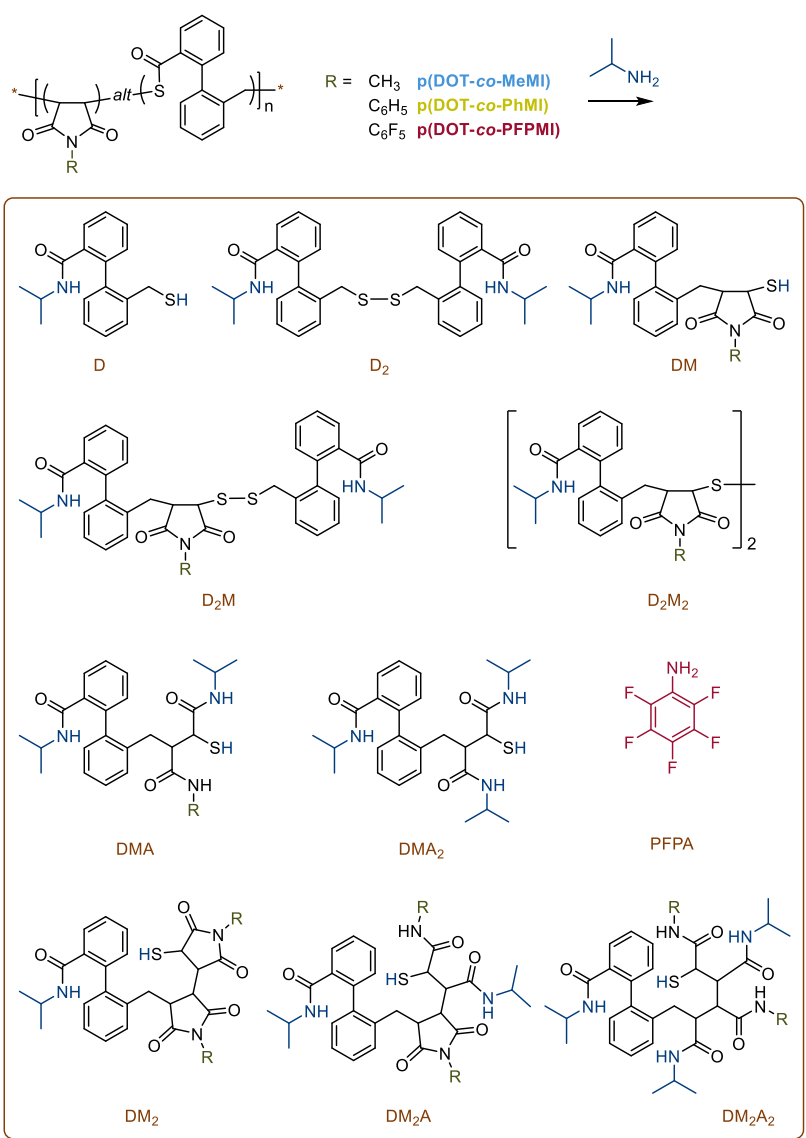

Scheme 2. Structures of the investigated degradation fragments where D denotes a DOT repeat unit, $\mathrm{M}$ a maleimide repeat unit and $\mathrm{A}$ an aminolytic cleavage of a maleimide repeat unit. Note that for $\mathrm{DM}_{2} \mathrm{~A}$ only one structural isomer is shown. The structure shown for $\mathrm{DM}_{2} \mathrm{~A}_{2}$ assumes that the opening of a maleimide is faster than the amide substitution reaction on an already-opened maleimide. Fragments observed for each copolymer are compiled in Table 1. 
Table 1. List of degradation fragments with indication of the observed ion(s) for each copolymer. The abbreviations are defined in Scheme 2. ${ }^{a}$

\begin{tabular}{l|l|l|l}
$\mathbf{R}=$ & $\mathbf{M e}$ & $\mathbf{P h}$ & $\mathbf{P F P}$ \\
\hline $\mathbf{D}$ & {$[\mathrm{M}+\mathrm{H}]^{+}$} & {$[\mathrm{M}+\mathrm{H}]^{+}$} & {$[\mathrm{M}+\mathrm{H}]^{+}$} \\
& {$[\mathrm{M}+\mathrm{Na}]^{+}$} & {$[\mathrm{M}+\mathrm{Na}]^{+}$} & \\
& {$[\mathrm{M}+\mathrm{Cl}]^{-}$} & & \\
$\mathbf{D}_{2}$ & {$[\mathrm{M}+\mathrm{H}]^{+}$} & {$[\mathrm{M}+\mathrm{H}]^{+}$} & {$[\mathrm{M}+\mathrm{H}]^{+}$} \\
& {$[\mathrm{M}+\mathrm{Na}]^{+}$} & {$[\mathrm{M}+\mathrm{Na}]^{+}$} & {$[\mathrm{M}+\mathrm{Na}]^{+}$} \\
$\mathbf{D M}$ & {$[\mathrm{M}+\mathrm{H}]^{+}$} & {$[\mathrm{M}+\mathrm{Na}]^{+}$} & {$[\mathrm{M}+\mathrm{H}]^{+}$} \\
& {$[\mathrm{M}+\mathrm{Na}]^{+}$} & {$[\mathrm{M}+\mathrm{Cl}]^{-}$} & {$[\mathrm{M}+\mathrm{Na}]^{+}$} \\
& {$\left[\mathrm{M}+\mathrm{NH}_{4}\right]^{+}$} & & {$[\mathrm{M}-\mathrm{H}]^{-}$} \\
& {$[\mathrm{M}-\mathrm{H}]^{-}$} & & \\
& {$\left[\mathrm{M}+\mathrm{NO}_{3}\right]^{-}$} & & \\
$\mathbf{D}_{2} \mathbf{M}$ & {$[\mathrm{M}+\mathrm{H}]^{+}$} & n.d. & n.d. \\
& {$[\mathrm{M}+\mathrm{Na}]^{+}$} & & \\
$\mathbf{D}_{2} \mathbf{M}_{2}$ & {$[\mathrm{M}+\mathrm{Na}]^{+}$} & n.d. & n.d. \\
$\mathbf{D M A}^{b}$ & {$[\mathrm{M}+\mathrm{H}]^{+}$} & {$[\mathrm{M}+\mathrm{H}]^{+}$} & {$[\mathrm{M}+\mathrm{H}]^{+}$} \\
& {$[\mathrm{M}-\mathrm{H}]^{-}$} & {$[\mathrm{M}+\mathrm{Na}]^{+}$} & {$[\mathrm{M}-\mathrm{H}]^{-}$} \\
$\mathbf{D M A}_{\mathbf{2}}$ & n.d. & {$[\mathrm{M}+\mathrm{Na}]^{+}$} & {$[\mathrm{M}+\mathrm{H}]^{+}$} \\
$\mathbf{D M}_{2}$ & {$[\mathrm{M}+\mathrm{NH}]_{4}^{+}$} & {$[\mathrm{M}+\mathrm{H}]^{+}$} & n.d. \\
$\mathbf{D M}_{2} \mathbf{A}$ & n.d. & {$[\mathrm{M}+\mathrm{H}]^{+}$} & n.d. \\
$\mathbf{D M}_{2} \mathbf{A}_{2}$ & n.d. & {$[\mathrm{M}+\mathrm{H}]^{+}$} & n.d. \\
$\mathbf{P F P A}$ & - & - & {$[\mathrm{M}+\mathrm{H}]^{+}$} \\
& & & {$[\mathrm{M}-\mathrm{H}]^{-}$} \\
& & &
\end{tabular}

${ }^{a}$ Disulfides containing aminolysed maleimide groups were not detected (not included in this table and in Scheme 2).

${ }^{b}$ This fragment was not detected in positive or negative ion mode. 
Finally, ${ }^{19}$ F NMR spectroscopy was used to demonstrate the versatility of backbone thioesters. A spectrum of the intact copolymer p(DOT-alt-PFPMI) is shown in Figure 6A. After degradation with $N$-isopropylamine the spectrum, Figure 6B, showed sharp signals as to be expected for small molecules, including signals associated with 2,3,4,5,6-pentafluoroaniline, in agreement with the mass spectrometric results. The presence of a multitude of signals was presumed to result from the stereochemistry of the former backbone carbons and the formation of disulfides. In addition to aminolysis, thioesters are also susceptible to thiolysis. Treatment of p(DOT-alt-PFPMI) with npropanethiol in the presence of DBU as base (to form the reactive thiolate species) resulted in backbone degradation through transthioesterification. This reaction was selective to the thioester with no observed evidence of maleimide attack. However, the ${ }^{19}$ F NMR spectrum, Figure $6 \mathrm{C}$, showed characteristic signals associated with fluoride (as DBU hydrofluoride) and of $S$-functional S-tetrafluorophenyl thioethers formed through thiol-para-fluoro nucleophilic aromatic substitution. Thiol-para-fluoro substitution can proceed with click-like efficiency ${ }^{35}$ on certain pentafluorophenyl derivatives and has been attracting increasing attention from the synthetic polymer and material communities. ${ }^{36-38}$ Herein, the para-fluoro substitution was slower than the transthioesterification but served to demonstrate that the degradation conditions of DOT-maleimide copolymers can strongly influence the chemical nature of the degradation fragments. 

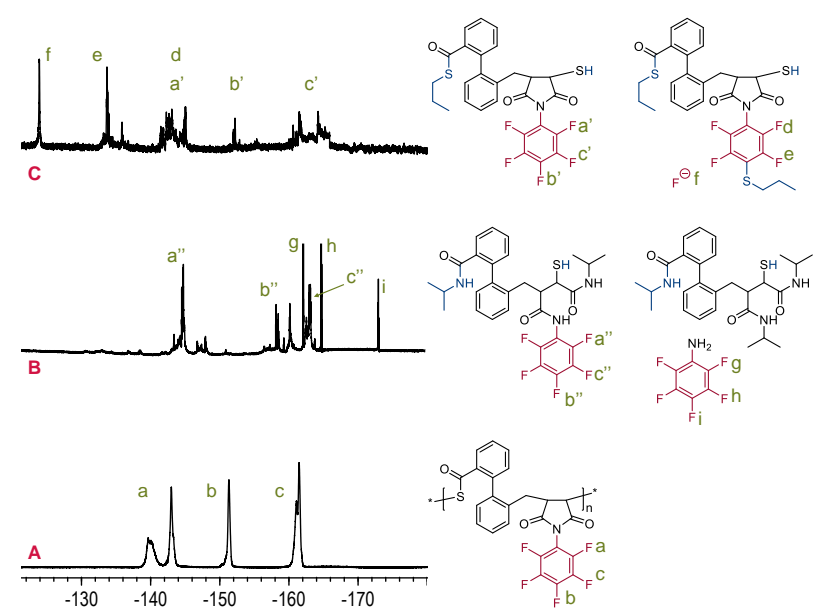

Figure 6. ${ }^{19} \mathrm{~F}$ NMR spectra of p(DOT-alt-PFPMI) (A) intact, showing a splitting of the ortho signals presumably due to tacticity; ${ }^{39}$ (B) after degradation with isopropylamine showing sharp signals including those associated with 2,3,4,5,6-pentafluoroaniline formed through double substitution on the maleimide ring; and (C) after degradation with n-propanethiol showing sharp signals including those characteristic of thiol-para-fluoro substitution on the side group.

\section{Conclusion}

Free radical homopolymerization of the thionolactone DOT produced a fully degradable thioesterfunctional material but in low yield and without options for property tuning. The combination of DOT with $N$-functional maleimides, especially the sterically more demanding $N$-phenyl and $N$ 2,3,4,5,6-pentafluorophenyl species, on the other hand, led to predominantly alternating copolymers able to degrade into fragments of predictable size while featuring a tuneable $N$-functionality. This work highlights several key advantages of thionolactones over cyclic ketene acetals (the most commonly used RROP monomers) in addition to the documented stability at ambient conditions of the DOT monomer. ${ }^{28}$ Specifically, when using $N$-phenylmaleimide and $N$-2,3,4,5,6pentafluorophenylmaleimide as comonomers, defects in the alternating sequences were mainly caused by a faster DOT incorporation leading to degradable DOT-DOT sequences, rather than nondegradable maleimide-maleimide sequences. Furthermore, maleimides can undergo a cycloaddition 
side reaction with cyclic ketene acetals, which causes loss of both comonomers. ${ }^{23,40}$ While we observed undefined red and pink side products when heating AIBN, DOT, and $N$-phenylmaleimide or $N-2,3,4,5,6$-pentafluorophenylmaleimide in DMSO, copolymerization of these comonomers proceeded smoothly and rapidly without observed side reactions when using acetonitrile or anisole as solvents. Finally, backbone thioesters offer a wider variety of degradation options than oxoesters, which can involve concurrent modification of the maleimide repeat units or their side groups.

Though realised rather crudely here, the combination of differently reactive functional groups (here: thioesters, imides, and pentafluorophenyl) is a promising strategy to design "smart" degradable materials that have the ability to "respond" to different degradation conditions through the formation of specific degradation products. It is thus anticipated that the thiocarbonyl addition-ringopening radical polymerization of thionolactones may offer new perspectives in existing and emerging applications of polymers with tailored degradability.

\section{Acknowledgements}

The authors acknowledge the University of Surrey Sustainability Research Theme for a summer bursary for M.P.S, the Department of Chemistry for a studentship for N.M.B., and Violeta Dukova (Surrey) for measuring DSC. Mass spectrometric analysis was enabled by an EPSRC strategic equipment grant (EP/P001440/1) and an EPSRC fellowship (EP/R031118/1).

Supperting Information. Experimental section, NMR spectra of DOT homopolymer, estimation of DOT homopolymer glass transition temperature, NMR and FT-IR spectra of maleimide copolymers before and after degradation. 


\section{References}

1. Brannigan, R. P.; Dove, A. P. Synthesis, properties and biomedical applications of hydrolytically degradable materials based on aliphatic polyesters and polycarbonates. Biomaterials Science 2017, 5 (1), 9-21 DOI: 10.1039/C6BM00584E.

2. Delplace; Nicolas. Degradable vinyl polymers for biomedical applications. Nat Chem 2015, 7 (10), 771 DOI: 10.1038/nchem.2343.

3. Tardy; Nicolas; Gigmes; Lefay; Guillaneuf. Radical Ring-Opening Polymerization: Scope, Limitations, and Application to (Bio)Degradable Materials. Chem Rev 2017, 117 (3), 1319 DOI: 10.1021/acs.chemrev.6b00319.

4. Becker, G.; Wurm, F. R. Functional biodegradable polymers via ring-opening polymerization of monomers without protective groups. Chemical Society Reviews 2018, 47 (20), 7739-7782 DOI: $10.1039 / \mathrm{C} 8 \mathrm{CS} 00531 \mathrm{~A}$.

5. Bailey, J.; Chou, J. L.; Feng, P. Z.; Kuruganti, V.; Zhou, L. L. Recent advances in free radical ring-opening polymerization. Acta Polymerica 1988, 39 (7), 335-341 DOI: 10.1002/actp.1988.010390702.

6. Agarwal, S. Chemistry, chances and limitations of the radical ring-opening polymerization of cyclic ketene acetals for the synthesis of degradable polyesters. Polymer Chemistry 2010, 1 (7), 953-964 DOI: 10.1039/COPY00040J.

7. Evans; Moad; Rizzardo; Thang. New Free-Radical Ring-Opening Acrylate Monomers. Macromolecules 1994, 27 (26), 7935 DOI: 10.1021/ma00104a062.

8. Paulusse; Amir; Evans; Hawker. Free Radical Polymers with Tunable and Selective Bio- and Chemical Degradability. JACS 2009, 131 (28), 9805 DOI: 10.1021/ja903245p.

9. Phelan, M.; Aldabbagh, F.; Zetterlund, P. B.; Yamada, B. Mechanism and Kinetics of the Free Radical Ring-Opening Polymerization of Eight-Membered Cyclic Allylic Disulfide Monomers. Macromolecules 2005, 38 (6), 2143-2147 DOI: 10.1021/ma047894i.

10. Moad, G., RAFT Polymerization - Then and Now. In Controlled Radical Polymerization: Mechanisms, American Chemical Society: 2015; Vol. 1187, pp 211-246.

11. Ribelli, T. G.; Lorandi, F.; Fantin, M.; Matyjaszewski, K. Atom Transfer Radical Polymerization: Billion Times More Active Catalysts and New Initiation Systems. Macromolecular Rapid Communications 2019, 40 (1), 1800616 DOI: 10.1002/marc.201800616. 
12. Penfold, N. J. W.; Yeow, J.; Boyer, C.; Armes, S. P. Emerging Trends in PolymerizationInduced Self-Assembly. ACS Macro Letters 2019, 8 (8), 1029-1054 DOI: 10.1021/acsmacrolett.9b00464.

13. Thickett, S. C.; Teo, G. H. Recent advances in colloidal nanocomposite design via heterogeneous polymerization techniques. Polymer Chemistry 2019, 10 (23), 2906-2924 DOI: 10.1039/C9PY00097F.

14. Guégain, E.; Michel, J.-P.; Boissenot, T.; Nicolas, J. Tunable Degradation of Copolymers Prepared by Nitroxide-Mediated Radical Ring-Opening Polymerization and Point-by-Point Comparison with Traditional Polyesters. Macromolecules 2018, 51 (3), 724-736 DOI: 10.1021/acs.macromol.7b02655.

15. Ratcliffe, L. P. D.; Couchon, C.; Armes, S. P.; Paulusse, J. M. J. Inducing an Order-Order Morphological Transition via Chemical Degradation of Amphiphilic Diblock Copolymer Nano-Objects. Biomacromolecules 2016, 17 (6), 2277-2283 DOI: 10.1021/acs.biomac.6b00540.

16. Hedir, G. G.; Bell, C. A.; Ieong, N. S.; Chapman, E.; Collins, I. R.; O’Reilly, R. K.; Dove, A. P. Functional Degradable Polymers by Xanthate-Mediated Polymerization. Macromolecules 2014, 47 (9), 2847-2852 DOI: 10.1021/ma500428e.

17. Huang, J.; Gil, R.; Matyjaszewski, K. Synthesis and characterization of copolymers of 5,6benzo-2-methylene-1,3-dioxepane and n-butyl acrylate. Polymer 2005, 46 (25), 11698-11706 DOI: https://doi.org/10.1016/j.polymer.2005.09.048.

18. Folini, J.; Huang, C.-H.; Anderson, J. C.; Meier, W. P.; Gaitzsch, J. Novel monomers in radical ring-opening polymerisation for biodegradable and $\mathrm{pH}$ responsive nanoparticles. Polymer Chemistry 2019, 10 (39), 5285-5288 DOI: 10.1039/C9PY01103J.

19. Ko, J. H.; Terashima, T.; Sawamoto, M.; Maynard, H. D. Fluorous Comonomer Modulates the Reactivity of Cyclic Ketene Acetal and Degradation of Vinyl Polymers. Macromolecules 2017, 50 (23), 9222-9232 DOI: 10.1021/acs.macromol.7b01973.

20. Sun; Zhuo; Liu. Synthesis and enzymatic degradation of 2-methylene-1,3-dioxepane and methyl acrylate copolymers. J Polym Sci Pol Chem 2003, 41 (18), 2898 DOI: 10.1002/pola.10868.

21. Shi, Y.; Agarwal, S., Thermally stable optically transparent copolymers of 2-methylene-1,3dioxepane and N-phenyl maleimide with degradable ester linkages. In e-Polymers, 2015; Vol. $15, \mathrm{p} 217$. 
22. Hill, M. R.; Guégain, E.; Tran, J.; Figg, C. A.; Turner, A. C.; Nicolas, J.; Sumerlin, B. S. Radical Ring-Opening Copolymerization of Cyclic Ketene Acetals and Maleimides Affords Homogeneous Incorporation of Degradable Units. ACS Macro Letters 2017, 6 (10), 10711077 DOI: 10.1021/acsmacrolett.7b00572.

23. Hill, M. R.; Kubo, T.; Goodrich, S. L.; Figg, C. A.; Sumerlin, B. S. Alternating Radical RingOpening Polymerization of Cyclic Ketene Acetals: Access to Tunable and Functional Polyester Copolymers. Macromolecules 2018, 51 (14), 5079-5084 DOI: 10.1021/acs.macromol.8b00889.

24. Huang, J.; Turner, S. R. Recent advances in alternating copolymers: The synthesis, modification, and applications of precision polymers. Polymer 2017, 116, 572-586 DOI: https://doi.org/10.1016/j.polymer.2017.01.020.

25. Mayo, F. R.; Walling, C. Copolymerization. Chemical Reviews 1950, 46 (2), 191-287 DOI: $10.1021 / \mathrm{cr} 60144 \mathrm{a} 001$.

26. Hisano, M.; Takashima, T.; Jin, Z.; Shiibashi, A.; Matsumoto, A. Radical Copolymerization Reactivity of N-Substituted Maleimides with $\alpha$-Substituted Styrenes with Various N- and $\alpha$ Substituents and the Thermal and Optical Properties of the Resulting Copolymers. Macromolecular Chemistry and Physics 2013, 214 (14), 1612-1620 DOI: 10.1002/macp.201300228.

27. Tawney, P. O.; Snyder, R. H.; Conger, R. P.; Leibbrand, K. A.; Stiteler, C. H.; Williams, A. R. The Chemistry of Maleimide and Its Derivatives. II. Maleimide and N-Methylolmaleimide. The Journal of Organic Chemistry 1961, 26 (1), 15-21 DOI: 10.1021/jo01060a004.

28. Bingham, N. M.; Roth, P. J. Degradable vinyl copolymers through thiocarbonyl addition-ringopening (TARO) polymerization. Chem Commun (Camb) 2019, 55 (1), 55-58 DOI: $10.1039 / \mathrm{c} 8 \mathrm{cc} 08287 \mathrm{a}$.

29. Smith, R. A.; Fu, G.; McAteer, O.; Xu, M.; Gutekunst, W. R. Radical Approach to ThioesterContaining Polymers. Journal of the American Chemical Society 2019, 141 (4), 1446-1451 DOI: $10.1021 /$ jacs.8b12154.

30. Bannin, T. J.; Kiesewetter, M. K. Poly(thioester) by Organocatalytic Ring-Opening Polymerization. Macromolecules 2015, 48 (16), 5481-5486 DOI: 10.1021/acs.macromol.5b01463. 
31. Datta, P. P.; Kiesewetter, M. K. Controlled Organocatalytic Ring-Opening Polymerization of $\varepsilon$-Thionocaprolactone. Macromolecules 2016, 49 (3), 774-780 DOI:

10.1021/acs.macromol.6b00136.

32. Nishimori, K.; Sawamoto, M.; Ouchi, M. Design of maleimide monomer for higher level of alternating sequence in radical copolymerization with styrene. Journal of Polymer Science Part A: Polymer Chemistry 2019, 57 (3), 367-375 DOI: 10.1002/pola.29191.

33. Doi, T.; Akimoto, A.; Matsumoto, A.; Otsu, T. Radical copolymerization of Nalkylmaleimides with isobutene and the properties of the resulting alternating copolymers. Journal of Polymer Science Part A: Polymer Chemistry 1996, 34 (3), 367-373 DOI: 10.1002/(SICI)1099-0518(199602)34:3<367::AID-POLA5>3.0.CO;2-R.

34. Liu, J.; Wang, H.; Manicke, N. E.; Lin, J.-M.; Cooks, R. G.; Ouyang, Z. Development, Characterization, and Application of Paper Spray Ionization. Analytical Chemistry 2010, 82 (6), 2463-2471 DOI: 10.1021/ac902854g.

35. Noy, J.-M.; Koldevitz, M.; Roth, P. J. Thiol-reactive functional poly(meth)acrylates: multicomponent monomer synthesis, RAFT (co)polymerization and highly efficient thiolpara-fluoro postpolymerization modification. Polym. Chem. 2015, 6 (3), 436-447 DOI: 10.1039/c4py01238k.

36. Agar, S.; Baysak, E.; Hizal, G.; Tunca, U.; Durmaz, H. An emerging post-polymerization modification technique: The promise of thiol-para-fluoro click reaction. Journal of Polymer Science Part A: Polymer Chemistry 2018, 56 (12), 1181-1198 DOI: doi:10.1002/pola.29004.

37. Delaittre, G.; Barner, L. The para-fluoro-thiol reaction as an efficient tool in polymer chemistry. Polymer Chemistry 2018, 9 (20), 2679-2684 DOI: 10.1039/C8PY00287H.

38. Bhebhe, M. N.; De Eulate, E. A.; Pei, Y.; Arrigan, D. W. M.; Roth, P. J.; Lowe, A. B. Reactive Conjugated Polymers: Synthesis, Modification, and Electrochemical Properties of Polypentafluorophenylacetylene (Co)Polymers. Macromolecular Rapid Communications 2017, 38 (2), 1600450-n/a DOI: 10.1002/marc.201600450.

39. Noy, J.-M.; Friedrich, A.-K.; Batten, K.; Bhebhe, M. N.; Busatto, N.; Batchelor, R. R.; Kristanti, A.; Pei, Y.; Roth, P. J. Para-Fluoro Postpolymerization Chemistry of Poly(pentafluorobenzyl methacrylate): Modification with Amines, Thiols, and Carbonylthiolates. Macromolecules 2017, 50 (18), 7028-7040 DOI: 10.1021/acs.macromol.7b01603. 
40. Hall, H. K.; Padias, A. B. Bond Forming Initiation of "Charge-Transfer" Polymerizations and the Accompanying Cycloadditions. Accounts of Chemical Research 1997, 30 (8), 322-329 DOI: 10.1021/ar950222f. 


\section{For Table of Contents Use Only}

Fully Degradable Thioester-functional Homo- and Alternating Copolymers Prepared through Thiocarbonyl Addition-Ring-opening RAFT Radical Polymerization

Matt P. Spick, Nathaniel M. Bingham, Yuman Li, Janella de Jesus, Catia Costa, Melanie J. Bailey, Peter J.

Roth

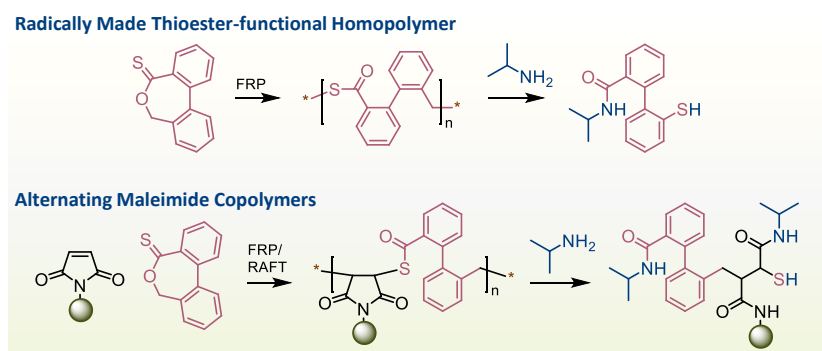

